\title{
Electron quantization in arbitrarily shaped gold islands on MgO thin films
}

\author{
Christian Stiehler, ${ }^{1}$ Yi Pan, ${ }^{1}$ Wolf-Dieter Schneider, ${ }^{1,2}$ Pekka Koskinen, ${ }^{3}$ Hannu Häkkinen, ${ }^{3,4}$ \\ Niklas Nilius, ${ }^{1,5},{ }^{*}$ and Hans-Joachim Freund ${ }^{1}$ \\ ${ }^{1}$ Fritz-Haber-Institut der Max-Planck-Gesellschaft, Faradayweg 4-6, 14195 Berlin, Germany \\ ${ }^{2}$ Ecole Polytechnique Federale de Lausanne, Institute of Condensed Matter Physics, CH-1015 Lausanne, Switzerland \\ ${ }^{3}$ Department of Physics, Nanoscience Center, University of Jyväskylä, P.O. Box 35 (YFL), FI-40014 Jyväskylä, Finland \\ ${ }^{4}$ Department of Chemistry, Nanoscience Center, University of Jyväskylä, P.O. Box 35 (YFL), FI-40014 Jyväskylä, Finland \\ ${ }^{5}$ Carl von Ossietzky Universität Oldenburg, Institut für Physik, D-26111 Oldenburg, Germany
}

(Received 5 July 2013; published 9 September 2013)

\begin{abstract}
Low-temperature scanning tunneling microscopy has been employed to analyze the formation of quantum well states (QWS) in two-dimensional gold islands, containing between 50 and 200 atoms, on $\mathrm{MgO}$ thin films. The energy position and symmetry of the eigenstates are revealed from conductance spectroscopy and imaging. The majority of the QWS originates from overlapping Au 6p orbitals in the individual atoms and is unoccupied. Their characteristic is already reproduced with simple particle-in-a-box models that account for the symmetry of the islands (rectangular, triangular, or linear). However, better agreement is achieved when considering the true atomic structure of the aggregates via a density functional tight-binding approach. Based on a statistically relevant number of single-island data, we have established a correlation between the island geometry and the gap between the highest-occupied and the lowest-unoccupied molecular orbital in the finite-sized islands. The linear eccentricity is identified as a suitable descriptor for this relationship, as it combines information on both island size and island shape. Finally, the depth of the confinement potential is determined from the spatial extension of QWS beyond the physical boundaries of the Au islands. Our paper demonstrates how electron quantization effects can be analyzed in detail in metal nanostructures. The results may help elucidating the interplay between electronic and chemical properties of oxide-supported clusters as used in heterogeneous catalysis.
\end{abstract}

DOI: 10.1103/PhysRevB.88.115415 PACS number(s): 73.21.La, 68.47.Gh, 68.37.Ef, 71.15.Mb

\section{INTRODUCTION}

Electron quantization is a fundamental phenomenon that accompanies the transition from bulk metals to small aggregates and down to single atoms. ${ }^{1}$ It occurs when the spatial dimension of an electron system reaches the Fermi wavelength and the electrons start to feel the boundaries of the system. At this stage, the quasicontinuous electronic structure of the bulk breaks apart into a set of discrete electronic states that are no longer defined by distinct wave vectors, as the translational symmetry of the system is lost. ${ }^{2-5}$ The energy gaps that open up between these quantum well states (QWS) depend approximately on the inverse atom number; however, this relationship is nonmonotonous, so the term "nonscalable" size regime was coined to describe the transition. ${ }^{6,7}$ Apart from the atom number, the symmetry of the confinement potential is the most important factor for the electronic structure of a nanoparticle. In simple terms, compact three-dimensional (3D) clusters feature a large overlap between the individual, atomic wavefunctions and therefore develop a metallic ground state more quickly than nonspherical aggregates do. Conversely, highly symmetric clusters are characterized by a large degeneracy of their electronic states, accompanied by the opening of gaps in the state density. Following this argument, the level splitting should be smaller in nonsymmetric aggregates, where the level degeneracy is lifted. In general, the critical size at which the nonmetal-to-metal transition occurs in a nanostructure results from the interplay of various, sometimes counteracting effects, among which the atom count and crystallographic order, the symmetry of the confinement potential, and shell-closing as well as surface phenomena are the most relevant. ${ }^{1,8}$ Consequently, the electronic ground state of a metal particle is difficult to predict in the nonscalable size regime even with $a b$ initio calculations.

Electron quantization effects have been comprehensively studied in gas-phase experiments, concentrating mainly on size-selected clusters with less than 100 atoms. ${ }^{9}$ However, experiments on supported clusters are hampered by two fundamental problems: (1) the unavoidable size and shape distribution of deposits in an ensemble and (2) their electronic coupling to the substrate. The first issue may be solved by the application of scanning probe techniques, e.g., scanning tunneling microscopy (STM), which enable the exploration of individual deposits independent of the actual ensemble properties. With this approach, quantization effects have been analyzed for a variety of confinement potentials, including linear, ${ }^{10,11}$ triangular, ${ }^{12}$ square, ${ }^{13}$ circular, $^{14-16}$ and hexagonal ${ }^{17}$ wells, as well as 3D boxes. ${ }^{3,18}$ While analytic solutions of the Schrödinger equation are available for simple geometries, numerical approaches need to be employed to study complex and partly disordered potential wells. ${ }^{19}$ Electronic decoupling, on the other hand, can be achieved by choosing suitable substrates for the experiment. Whereas metal supports always give rise to certain interfacial interactions, ${ }^{11,13,14,17}$ nearly complete electronic separation is possible with wide-gap substrates. The latter approach works best when using thin insulating films grown on metal surfaces, as such systems retain a residual conductivity and are therefore insensitive to charging in electron- and ion-mediated experiments. ${ }^{20}$ The versatility of the thin film approach has been demonstrated for a large number of studies on metal particles supported on oxide $\left(\mathrm{Al}_{2} \mathrm{O}_{3}, \mathrm{MgO}, \mathrm{CaO}, \mathrm{TiO}_{2}\right.$, and $\left.\mathrm{CeO}_{2}\right)$, halide $(\mathrm{NaCl}$ and $\mathrm{KBr})$, and sulfide $\left(\mathrm{MoS}_{2}\right)$ surfaces. ${ }^{5,21-24}$ 
The elucidation of quantization effects in ultrasmall metal particles is not of academic interest only. In heterogeneous catalysis, the transition from insulating to metallic behavior has been associated with the onset of catalytic activity in the metal deposits. ${ }^{7,25}$ Gold clusters on $\mathrm{TiO}_{2}$, for example, show high reactivity in the low-temperature $\mathrm{CO}$ oxidation as long as they feature a gap between the highest-occupied and lowest-unoccupied molecular orbital (HOMO-LUMO). ${ }^{26}$ The onset of metallicity is also of relevance for the optical properties of nanostructures, which evolve from single-electron to plasmonic excitations with an increasing number of atoms in the system. ${ }^{27}$

In this paper, we study the electronic structure of differently shaped, two-dimensional (2D) Au islands grown on $\mathrm{MgO} / \mathrm{Ag}(001)$ thin films. Typical islands contain between 50 and 200 atoms, covering the critical size range in which the metal-insulator transition occurs in gold. The development of QWS is investigated with low-temperature tunneling microscopy and spectroscopy. The experimental symmetry and energy position of the QWS are compared to simple analytical models and density functional tight-binding $(\text { DFTB })^{28}$ calculations that account for details in the island structure. The extensive acquisition of single-cluster data allows us to follow the evolution of the HOMO-LUMO gap with atom count and island symmetry. On this basis, we draw general conclusions on the interplay between geometric and electronic properties of supported gold nanostructures.

\section{EXPERIMENT AND THEORY}

The experiments have been performed with a custom-built, helium-cooled STM operated at ultra-high-vacuum conditions. Whereas morphological surface information was generated with constant-current scans, state-density data were derived from differential conductance $(d I / d V)$ spectra acquired with a lock-in technique. For this purpose, the tip was positioned above a selected metal island and the current derivative was detected as a function of bias voltage. According to the Tersoff-Hamann theory for vacuum tunneling, ${ }^{29}$ the $d I / d V$ versus $V$ curves provide a measure of the local density of states (LDOS) at the sample surface. The Au islands investigated here have been deposited onto monolayer- (ML) and bilayer-thick $\mathrm{MgO}$ films grown on a $\mathrm{Ag}(001)$ support. ${ }^{30}$ The films were prepared by reactive $\mathrm{Mg}$ deposition in $5 \times 10^{-7} \mathrm{mbar}_{2}$ onto the sputtered and annealed silver surface at $550 \mathrm{~K}$. The film quality was checked with low energy electron diffraction and STM, revealing the sharp $(1 \times 1)$ square pattern of the $(001)$ rocksalt plane in diffraction and extended, atomically flat oxide terraces in microscopy [Fig. 1(a)]. The ultrathin nature of the film was verified with $d I / d V$ spectra, exhibiting a distinct shoulder at $1.7 \mathrm{~V}$ inside the $\mathrm{MgO}$ band gap [Fig. 1(b)]. The peak originates from a $\mathrm{Ag}-\mathrm{MgO}$ interface state that splits off the oxide conduction band and is detected only in the limit of ML films. ${ }^{30}$ Gold with a nominal thickness of 0.05 ML was deposited from a Au-coated tungsten filament onto the sample held at $15 \mathrm{~K}$. To promote atom diffusion and particle formation, the sample was annealed inside the STM to $100 \mathrm{~K}$. The resulting aggregates contained 50-200 atoms in average and grew exclusively in the form of ML sheets. ${ }^{31}$ This unusual 2D growth regime has been explained with a charge transfer from
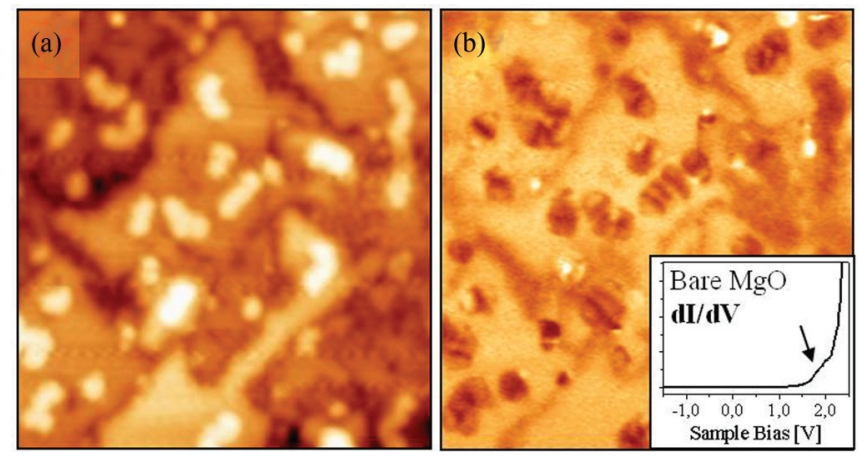

FIG. 1. (Color online) (a) STM topographic image and (b) corresponding $d I / d V$ map of $2 \mathrm{ML} \mathrm{MgO/Ag} \mathrm{(001)} \mathrm{covered} \mathrm{with}$ ML Au islands $\left(50 \times 50 \mathrm{~nm}^{2}, 1.7 \mathrm{~V}, 100 \mathrm{pA}\right)$. The inset in (b) shows a $d I / d V$ spectrum of the bare oxide. Whereas the sharp rise at $2.5 \mathrm{~V}$ marks the onset of the $\mathrm{MgO}$ conduction band, the small shoulder at $1.7 \mathrm{~V}$ is due to an $\mathrm{MgO} / \mathrm{Ag}(001)$ interface state (arrow).

the Ag substrate through the $\mathrm{MgO}$ spacer into the $\mathrm{Au}$ islands. This electron exchange is a cooperative effect between the low $\mathrm{MgO} / \mathrm{Ag}$ workfunction and the high electronegativity of gold. ${ }^{32}$ The resulting Au anions experience enhanced bonding to the oxide film, which is mediated by Coulomb and polaronic interactions with the $\mathrm{MgO}$ surface and renders a 2D growth energetically preferred. ${ }^{33}$ In contrast, formation of $3 \mathrm{D}$ particles prevails on most bulk oxides due to the small metal-oxide adhesion in that case.

To establish a correspondence between experiment and theory, we have analyzed the electronic structure of $\mathrm{Au}$ islands by DFTB calculations. ${ }^{28}$ Our approach provides a reasonable description of metallic states in $2 \mathrm{D}$ and $3 \mathrm{D}$ clusters and is therefore suited to describe even extended metal nanostructures. ${ }^{34}$ Using the atomic configuration deduced from STM, we have treated the Au sheets as free-standing islands and included the substrate only via a charge transfer of 0.2 e/atom to account for the electron tunneling from the $\mathrm{MgO} / \mathrm{Ag}$ support into the gold. ${ }^{5,21}$ The disregard of other metal-oxide coupling mechanisms, such as interfacial hybridization, is justified, as no direct chemical bonds are formed between the gold and the oxide surface. To render the DFTB calculations comparable to the STM data, we have placed the tight-binding wavefunctions onto a spatial grid and performed state-resolved STM simulations. The final states that dominate STM tunneling at positive bias were identified as the $6 p_{z}$ orbitals of gold. The experimental results can now be reproduced by mapping the Mulliken population of the $6 p_{z}$ states onto the island shape, an approach that was proven to give fair agreement with experimental STM images in previous papers. $^{21}$

\section{RESULTS AND DISCUSSION}

In the following paragraphs, we present three model islands with rectangular, triangular, and quasilinear symmetry. On the basis of these sample cases, we discuss general issues of electron quantization in gold nanostructures at the end of this paper. 
(a)

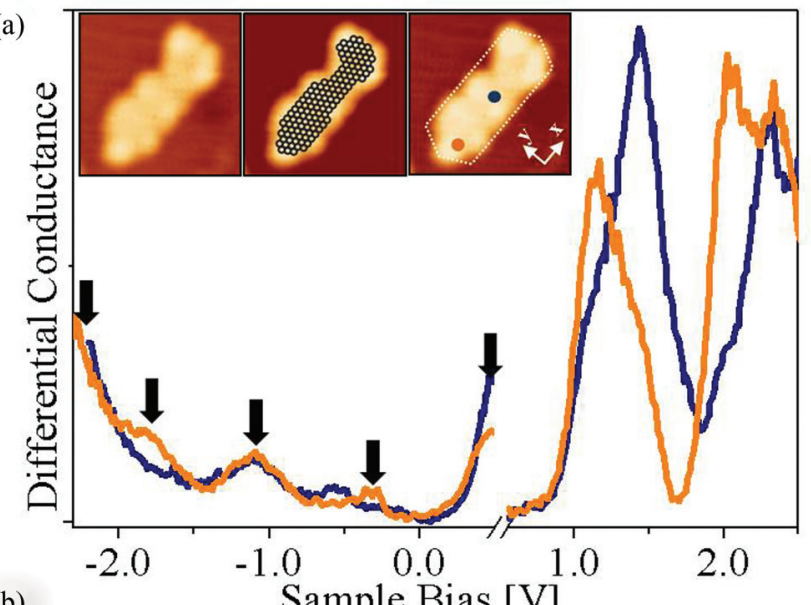

(b)

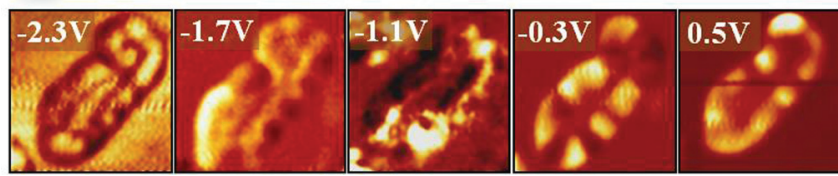

FIG. 2. (Color online) (a) Differential conductance spectra taken in the center (blue) and in the lower part (orange) of the rectangular $\mathrm{Au}$ island shown in the inset (setpoints: -2.0 and $+2.5 \mathrm{~V}$ for negative and positive polarity, respectively). The insets (from left to right) show a topographic image, a structure model, and the quasirectangular outline of the island $\left(7.5 \times 7.5 \mathrm{~nm}^{2},-0.1 \mathrm{~V}, 250 \mathrm{pA}\right)$. (b) Filledstate $d I / d V$ maps of the islands in (a). The selected bias voltages are indicated by arrows directly in the $d I / d V$ spectra. Note the pronounced localization of the state density at the cluster perimeter.

\section{A. Rectangular Au islands}

The first example discussed here is a nearly rectangular island $75 \AA$ in length and $30 \AA$ in width that contains $\sim 130$ atoms (Fig. 2). A structure model of the island is obtained by filling its circumference with a hexagonal close-packed arrangement of $\mathrm{Au}$ atoms, using the lattice parameter of bulk gold (2.89 $\AA$ ). A 5- $\AA$-wide rim is kept open along the perimeter to account for convolution effects of the STM tip. Uncertainties in this procedure limit the accuracy of our structure determination and introduce a $20 \%$ error in the total atom count, which corresponds to roughly one atom row at the perimeter.

The valence electronic structure of the Au island has been investigated via $d I / d V$ spectroscopy and mapping and yields the energy position and spatial localization of its QWS. Two $d I / d V$ spectra taken in the lower and central parts of the island are depicted in Fig. 2(a). There is a pronounced difference between the negative-bias spectrum (filled states), which is rather flat, and the positive one, which displays pronounced empty-state resonances. At first glance, this difference reflects the lower tunneling probability out of filled states due to their larger spatial confinement as compared to empty ones. In addition, the islands are subject to a redistribution of the filled-state density, which is triggered by the electron transfer from the $\mathrm{MgO} / \mathrm{Ag}$ film to the $\mathrm{Au}$ electronic states discussed before. ${ }^{32}$ The extra charges that amount to $\sim 0.2|e|$ per interfacial Au atom, or 26 electrons for the total island, tend to localize along the island perimeter in order to minimize the internal electron repulsion. ${ }^{21,35}$ Consequently, the filled-state density is displaced from the island interior to its outer regions, and only small $d I / d V$ signals are detected in the center of the nanostructure.

Conversely, high intensity builds up at the island perimeter, as observed in $d I / d V$ maps taken around the Fermi level [Fig. 2(b)]. Over a relatively broad bias window, the edges of the negatively charged $\mathrm{Au}$ islands appear bright, indicating an enhancement of filled-state density at the perimeter. The spectral intensity becomes maximal at low-coordinated kink and corner sites, which are particularly suited to accommodate extra electrons because of their large distance to adjacent charge centers along the edge. No comparable effect is revealed for the empty states, which display no Coulomb repulsion and therefore no incentive for a charge-redistribution toward the edges. As the specific nature of edge states on negatively charged gold islands has been discussed before, ${ }^{21}$ we mainly focus on the empty-state spectra in this work.

The most prominent $d I / d V$ peaks are detected in the bias window between +1.0 and $+2.5 \mathrm{~V}$ [Fig. 2(a)], which matches the energy of the Au $6 p_{z}$ orbitals, according to density functional theory calculations. ${ }^{36}$ The $\mathrm{Au} 6 s$ manifold is mainly located below the Fermi level, while the few empty $6 s$ states are difficult to detect in STM due to their high orbital-momentum quantum numbers that give rise to a multitude of nodes in the orbital symmetry. ${ }^{14}$ For the rectangular island depicted in Fig. 2, at least six $d I / d V$ maxima have been detected at $1.05,1.25,1.45,1.7,2.0$, and $2.3 \mathrm{~V}$, all localized in the energy window of the Au $6 p_{z}$ states [Fig. 3(a)]. The energy position of the peaks is approximately constant across the island, reflecting their delocalized character. Their width amounts to $0.25 \mathrm{eV}$, a value that might be explained with the presence of vibronic sidebands dressing the actual electronic resonance. ${ }^{37}$

The intensity of the $d I / d V$ peaks was found to depend sensitively on the tip position above the island, in accordance with the nodal structure of the respective QWS. To analyze the symmetry of the underlying orbitals, we have acquired $d I / d V$ maps of the island over the entire bias range of interest [Fig. 3(b)]. The lowest $6 p_{z}$-type QWS at $1.05 \mathrm{~V}$ is characterized by a rather uniform $d I / d V$ intensity in the lower half of the island and a vanishing signal in the upper one. This intensity pattern reverses for the second state, when the upper half turns bright. The QWS situated at higher energy are always governed by nodal planes, i.e., by dark lines in the $d I / d V$ maps that cut through the island in the vertical direction and, at higher bias, in the horizontal direction. The third and fourth QWS, for example, display one and two vertical nodes, whereas the fifth and sixth states show one additional horizontal nodal plane [Fig. 3(b)].

A simple particle-in-the-box model is sufficient to reproduce the experimental sequence of QWS. In a first approximation, the island can be treated as rectangular box with infinite walls for which the wavefunctions and hence the quantization effects are separable along the two orthogonal axes $x$ and $y: E_{n_{x}, n_{y}}=E_{0}+\frac{\hbar^{2} \pi^{2}}{2 m_{\text {eff }} L_{x}^{2}}\left(n_{x}^{2}+c^{2} n_{y}^{2}\right){ }^{38}$ With the measured aspect ratio $c=L_{x} / L_{y}=2.5$, the eigenstates of the rectangular box may be fitted to the spectroscopy data [Fig. 4(a)], which yields an onset energy for the $\mathrm{Au} 6 p_{z}$ band of $E_{0}=0.75 \mathrm{eV}$ and an effective electron mass of $m_{\text {eff }}=0.18$ 
(a)

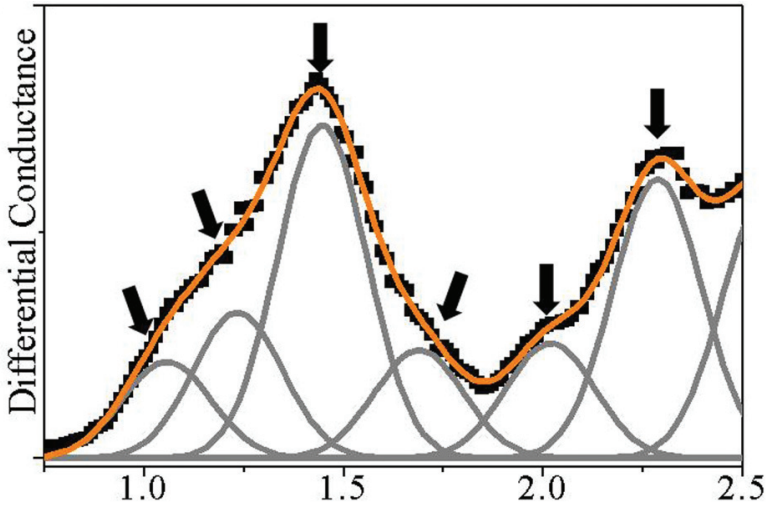

(b)
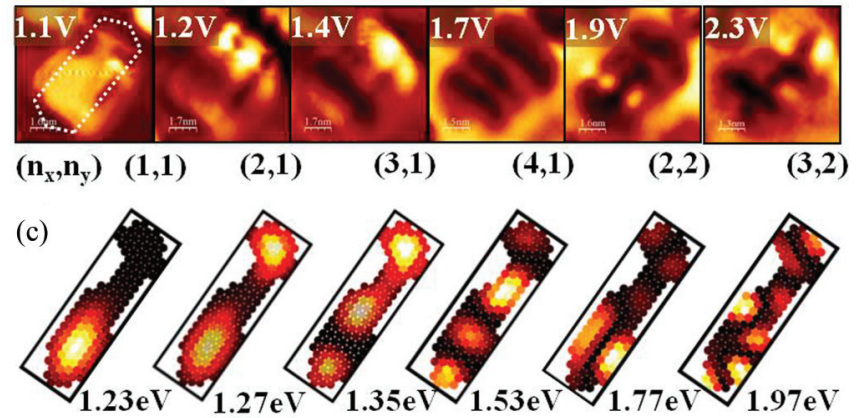

FIG. 3. (Color online) (a) Empty-state $d I / d V$ spectrum taken in the center of the rectangular island shown in Fig. 2. The spectral course is deconvoluted into Gaussians of constant width (gray), representing the individual QWS of the cluster. (b) Corresponding $d I / d V$ maps measured at the bias positions marked by arrows in (a) $\left(7.5 \times 7.5 \mathrm{~nm}^{2}\right)$. The symmetry of the QWS can be rationalized with a simple 2D particle-in-a-box model. (c) DFTB calculations showing the local-state density in the respective $\mathrm{Au}$ island. The second and third maps result from a superposition of two closely spaced eigenstates of the system.

$m_{e}$. The latter value matches the effective mass of the Au(111) surface state $\left(0.22 \mathrm{~m}_{\mathrm{e}}\right)^{39}$ but is smaller than $m_{\mathrm{eff}}$ in monatomic

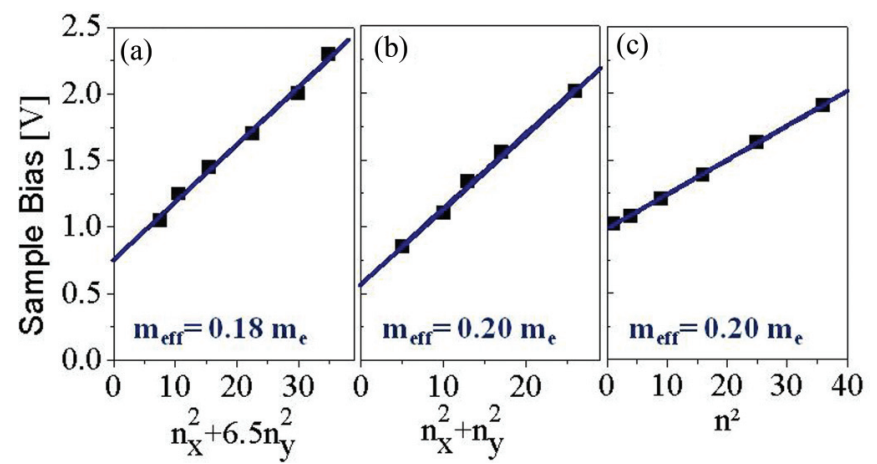

FIG. 4. (Color online) Dispersion relations for the (a) rectangular, (b) triangular, and (c) curved-linear cluster as deduced from the corresponding $d I / d V$ spectra. The measured bias values are plotted against the quantum numbers of the particle-in-a-box models with corresponding geometry. A linear fit through the data yields the effective electron mass and the onset energy of the underlying $\mathrm{Au}$ electronic states.
Au chains on $\operatorname{NiAl}(110)\left(0.5 \mathrm{~m}_{\mathrm{e}}\right) .{ }^{10}$ This discrepancy may be explained with the reduced dimensionality and the large interatomic spacing in one-dimensional (1D) gold chains.

In addition, the QWS symmetry predicted by the box model qualitatively matches the experimental results. In both simulation and experiment, the first four eigenstates are characterized by $0-3$ vertical nodes $\left(n_{x}=1,2,3,4\right)$, whereas the fifth and sixth experimental states are assigned to box states with one horizontal and two $(2,2)$ and three $(3,2)$ vertical nodes, respectively. However, this interpretation implies that two intermediate states described by $\left(n_{x}, n_{y}\right)=(1,2)$ and $(5,1)$ are not resolved in the experiment, most likely because of their overlap with neighboring QWS of different symmetry. Not surprisingly, a detailed analysis discloses substantial differences between measured $d I / d V$ maps and ideal box states, originating from inhomogeneities in the island potential. A better match between theory and experiment can thus be achieved if the low-energy QWS are calculated with the DFTB approach, using the atomic island configuration depicted in Fig. 2(a). ${ }^{28}$ The computed LDOS maps are able to reproduce the main features seen in experiment [Fig. 3(c)]. The first two eigenstates are confined to the lower and upper halves of the $\mathrm{Au}$ island, while the third state exhibits a pronounced vertical nodal plane. In addition, the fifth and sixth QWS are characterized by a first horizontal node in both simulation and experiment. The remaining discrepancies, even with the DFTB approach, are ascribed to irregularities in the island shape that are not captured in the atom models derived from our STM images. In particular, the edge configuration cannot be given in detail, although it may substantially alter the overall electronic structure of the island. Another aspect that has not been considered is the presence of defects in the $\mathrm{MgO}$ film, such as $\mathrm{O}$ vacancies. ${ }^{20} \mathrm{~A}$ better match between experiment and theory might be achieved with a more reliable structure determination, based on atomically resolved STM images of the nanoislands.

\section{B. Triangular Au islands}

A similar analysis has been carried out for an Au island of triangular shape and 55- $\AA$ edge length that contains $\sim 170$ atoms (Fig. 5). We have chosen this particular geometry, because an analytical solution of the Schrödinger equation exists for isosceles, right-angled triangular potentials, which enables us to model the QWS observed experimentally. ${ }^{40}$ The right-angled isosceles is the only triangular system that is fully separable in $x$ and $y$, as it can be treated as a square potential cut along its diagonal (with the boundary condition $n_{x} \neq n_{y}$ ). The similarity to the rectangular box also becomes evident from the analytical expression of the eigenenergies ${ }^{40}$ : $E_{n x}, n y=E_{0}+\frac{\hbar^{2} \pi^{2}}{2 m_{\mathrm{eff}} L_{x}^{2}}\left(n_{x}^{2}+n_{y}^{2}\right)$. Experimentally, six $d I / d V$ maxima at $0.9,1.05,1.2,1.55,1.75$ and $2.0 \mathrm{~V}$ have been detected in the energy range of the $\mathrm{Au} 6 p_{z}$ state manifold [Fig. 5(a)]. Fitting the peak energies to the dispersion relation of the triangular potential yields an onset energy of $E_{0}=0.6$ $\mathrm{eV}$ and an effective electron mass of $m_{\mathrm{eff}}=0.2 \mathrm{~m}_{\mathrm{e}}$, similar to the values obtained for the rectangular box. Moreover, the lowest eigenstates again feature well-defined symmetries, as seen in the respective $d I / d V$ maps [Fig. 5(b)]. While the ground state shows a homogeneous intensity distribution, two 


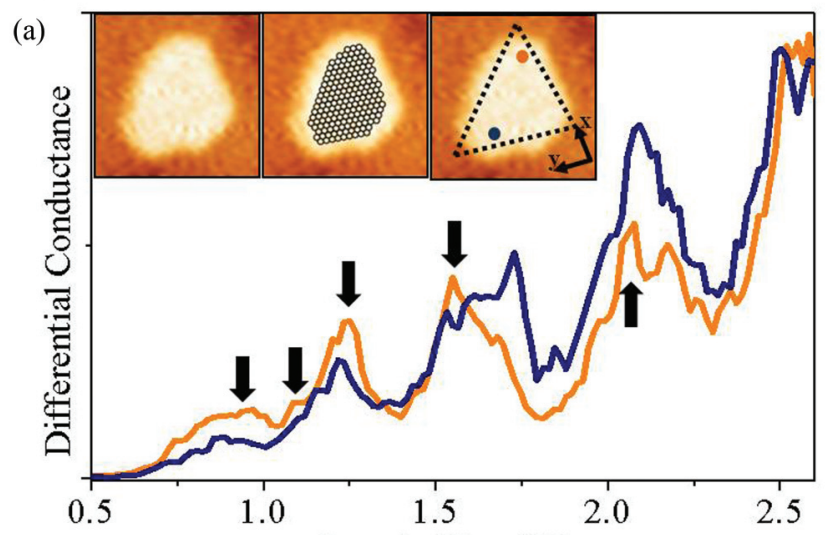

(b)

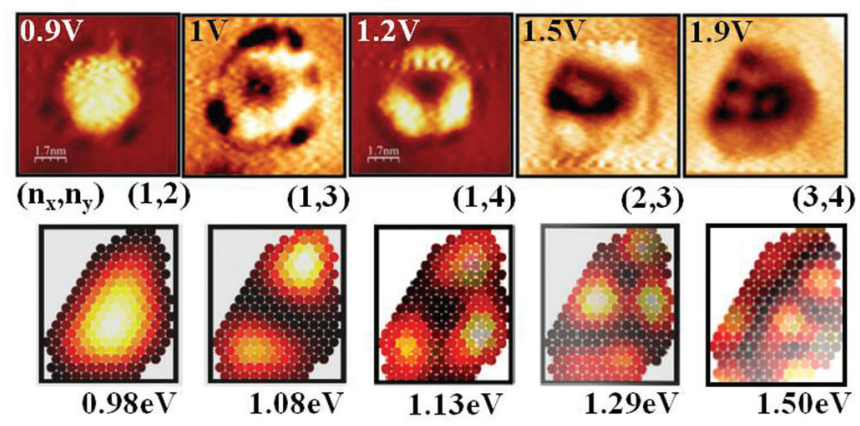

FIG. 5. (Color online) (a) Empty-state $d I / d V$ spectra taken in the upper (orange) and lower (blue) parts of the triangular island shown in the inset. Intensity maxima have been obtained by deconvoluting the curve into a set of Gaussians. (b) Corresponding $d I / d V$ maps taken at the peak positions marked by arrows in (a) $\left(8 \times 8 \mathrm{~nm}^{2}\right)$. The symmetry of the QWS can be explained either with the eigenstates of an isosceles, right-angled triangular potential or with DFTB calculations performed on a structure model of the Au island. Here, the symmetry of the third, fourth, and fifth $d I / d V$ maps result from a superposition of two adjacent eigenstates.

and three maxima are resolved for the second and third QWS. However, the fourth and fifth states show a more involved intensity pattern that is characterized by a complex sequence of dark and bright spots.

The respective LDOS patterns computed with the DFTB approach are shown in Fig. 5(b) for comparison. In particular, the lower QWS show a reasonable match to the $d I / d V$ maps, indicating the suitability of our theoretical method to capture the physics of electron quantization. The good correspondence underlines also the free-electron character of the gold QWS, which renders them insensitive to small irregularities in the potential.

\section{Linear Au islands}

The last island that is discussed in detail has an elongated structure and may be considered as a model for a quasilinear quantum well. Its main axis $(8.3 \mathrm{~nm})$ is roughly three times longer than its short one $(2.6 \mathrm{~nm})$, while the number of atoms has been approximated to be 130 (Fig. 6). Similar to the prior examples, several $d I / d V$ peaks are detected at positive bias, at 1.0, 1.1, 1.2, 1.4, and 1.9 V [Fig. 6(a)]. These energy values can be compared with the eigenstates (a)

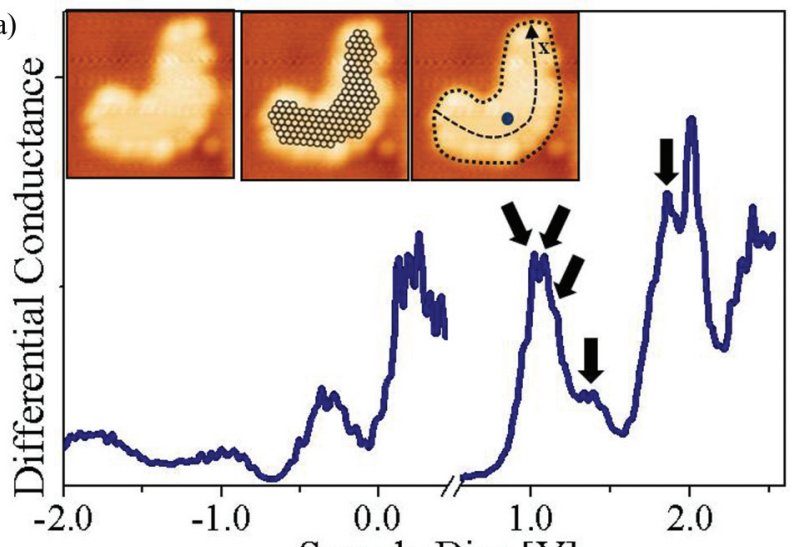

(b)

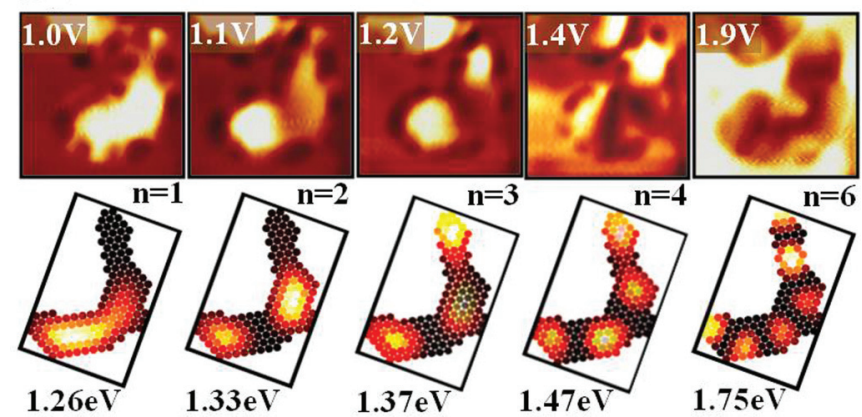

FIG. 6. (Color online) (a) Empty- and filled-state $d I / d V$ spectra taken in the center of the curved-linear island shown in the inset $\left(9 \times 9 \mathrm{~nm}^{2}\right)$. The first five maxima, obtained from a Gaussian deconvolution, are marked by arrows. (b) $d I / d V$ maps taken at the determined bias positions $\left(9 \times 9 \mathrm{~nm}^{2}\right)$. The symmetry of the QWS is compatible with those of a $1 \mathrm{D}$ particle-in-a-box model. The respective LDOS maps calculated with the DFTB method are shown below. The third and fourth maps are superpositions of two adjacent states.

of a $1 \mathrm{D}$ box potential that only depend on a single quantum number $n: E_{n}=E_{0}+\frac{\hbar^{2} \pi^{2}}{2 m_{\text {eff }} L^{2}} n^{2} \cdot{ }^{38}$ A least-mean-square fit to the $d I / d V$ data provides an onset energy of $E_{0}=1.0 \mathrm{eV}$ and an effective electron mass of $m_{\mathrm{eff}}=0.2 \mathrm{~m}_{\mathrm{e}}$ for the QWS manifold in this island. The density-probability distribution in a $1 \mathrm{D}$ box potential is described by charge-density waves with an increasing number of maxima and minima along the axis with increasing quantum number $n \cdot{ }^{38}$ Despite obvious deviations from a truly $1 \mathrm{D}$ potential, this scheme is still discernible in the experimental $d I / d V$ maps, at least for the lowest five QWS [Fig. 6(b)]. Whereas the first and second states show $d I / d V$ intensity mainly in the lower part of the island, the signal localizes in the upper part at around $1.2 \mathrm{~V}$ for the third QWS. With increasing bias, additional maxima and minima are detected along the main axis, although the pattern turns increasingly complex. No horizontal nodal planes becomes evident up to the highest bias investigated here, reflecting the pseudo-1D nature of the confinement potential. As stated before, a better reproduction of the experimental maps can be achieved when the atomic island configuration is taken into account using our DFTB method [Fig. 6(b)]. The corresponding LDOS maps clearly reveal the increasing number of nodal planes along the island axis with higher energy and higher quantum number $n$. 


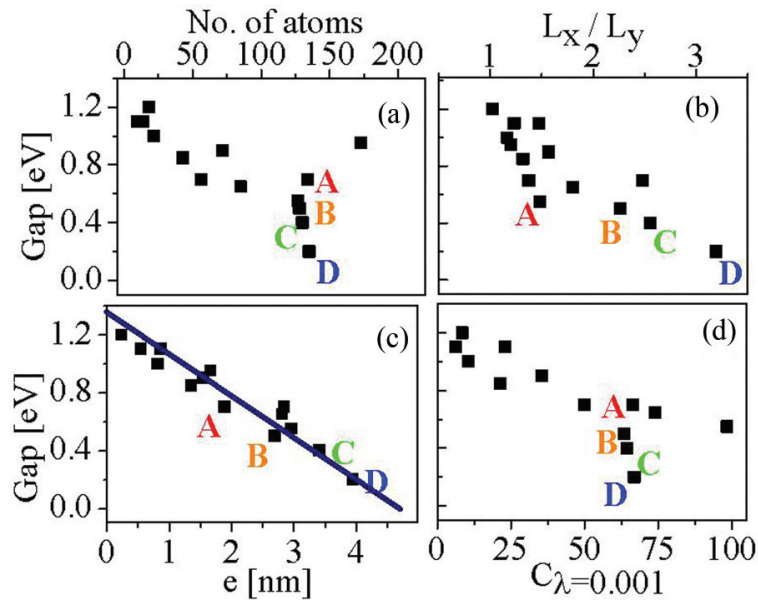

FIG. 7. (Color online) Size and shape dependence of the HOMOLUMO gap $\Delta$ plotted versus (a) the total number of atoms, (b) the ratio of semimajor and semiminor axis $L_{x} / L_{y}$, (c) the linear eccentricity $e$, and (d) the mean coordination number $c_{\lambda}=0.001$. To emphasize the shape effect on the gap size, four sample islands have been highlighted by letters (A-D). The corresponding islands are shown in Fig. 8. Note the good linear relationship between gap size and linear eccentricity in (c).

\section{General properties of QWS: HOMO-LUMO gap in arbitrarily shaped Au islands}

We now draw general conclusions on the interplay of geometry and electronic structure of planar $\mathrm{Au}$ islands on $\mathrm{MgO}(001)$ thin films, using the experimental results discussed before. A fundamental property of a confined electronic system is the energy gap $\Delta$ between the HOMO and the LUMO states, which provides a measure of its metallicity. ${ }^{6,8}$ To analyze this parameter, we have acquired $d I / d V$ spectra for $\sim 30$ $\mathrm{Au}$ islands and determined the region of zero conductance around the Fermi level. As expected, the gap size decreases with increasing island size, a trend that becomes evident when plotting the $d I / d V$ gap as a function of the estimated atom number $n$ per island [Fig. 7(a)]. For islands with less than 20 atoms, the onsets of filled and empty states are separated by more than $1.0 \mathrm{eV}$, a value that gradually decreases to 0 $\mathrm{V}$ for atom counts well above $100 .{ }^{5,41}$ Whereas the general size evolution is apparent, the data points exhibit a substantial scatter in Fig. 7(a) and cannot be described with a simple functional relationship. Evidently, the atom number is not the only parameter that governs the HOMO-LUMO gap and other properties, e.g., the island shape or nonmonotonous shell-closing effects, are of relevance as well. ${ }^{8}$

The shape effect can, in simple terms, be explained with the different degree of wavefunction overlap in compact clusters as compared to open, elongated nanostructures. In compact clusters, the number of interacting atoms in a fixed radius will be larger, which promotes rapid closing of the HOMO-LUMO gap. This trend might be counterbalanced by symmetry effects, as highly symmetric clusters feature a larger degeneracy of electronic states, which in turn inhibits gap closure. Symmetry effects are closely related to the presence of magic cluster sizes, being characterized by closed-shell configurations and pronounced HOMO-LUMO gaps. For gas-phase clusters, an oscillatory behavior of the gap has been revealed with size; however, similar experiments are missing for supported nanoparticles. ${ }^{8,42}$

To account for the interplay of size/shape and HOMOLUMO gap, we have searched for a suitable descriptor that catches the relevant geometric properties of the single-layer $\mathrm{Au}$ islands. A first choice has been the ratio of long and short axis, $L_{x} / L_{y}$, as a direct measure of the island shape. Plotting the HOMO-LUMO gap as a function of this parameter does not reduce the scatter in the data points with respect to the $\Delta-n$ diagrams [Fig. 7(b)]. However, as the axis ratio neglects the island size, a poor description of the HOMOLUMO gap is expected. Other shape parameters, e.g., the ratio between perimeter length and island area, do not improve this correlation either (not shown). In a next step, we tested the linear eccentricity $e=1 / 2 \sqrt{\left(L_{x}^{2}-L_{y}^{2}\right)}$, i.e., the square root of the difference between the squared island axes, as a descriptor for the HOMO-LUMO gap. The eccentricity contains both size and shape elements and has the dimension of length. Moreover, it is a common parameter to analyze gas-phase clusters. ${ }^{42}$ Plotting the HOMO-LUMO gap as a function of the eccentricity leads to an almost linear relationship, with little scatter in the data points [Fig. 7(c)]. The good correlation to the gap value $\Delta$ suggests that the eccentricity can be used to predict HOMO-LUMO gaps in metal islands. However, a clear disadvantage of the descriptor is its empirical character that lacks any insight into the quantum mechanical nature of the problem.

A microscopic parameter that may capture the origin of the gap is the atom coordination number, i.e., the number of first, second, third, etc., nearest-neighbor atom pairs in the island. Intuitively, one would expect that an increase in coordination enhances hybridization among the atoms and results in a more rapid crossover into a metallic ground state. To analyze this effect, we have calculated pair-correlation functions for all islands investigated here; four examples are shown in Fig. 8. The different shape of the model clusters becomes manifest in the tail of the correlation function, i.e., in the number of

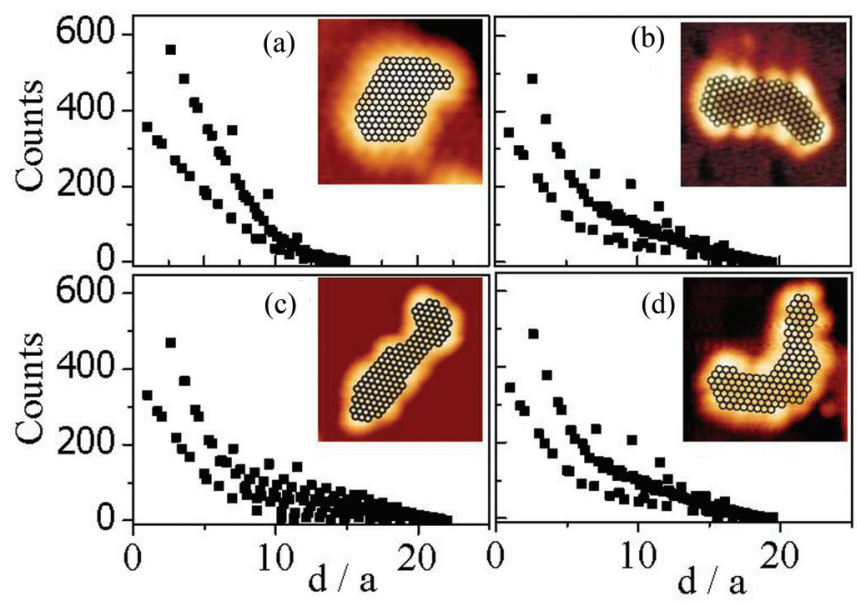

FIG. 8. (Color online) Pair correlation functions of four sample islands with similar atom counts $(\mathrm{A}=134, \mathrm{~B}=128, \mathrm{C}=130, \mathrm{D}=$ $135)$ but increasing eccentricity. Whereas the symmetric island shows a rapid decay, asymmetric clusters are characterized by pronounced tails in the pair-correlation function. The corresponding gap sizes can be deduced from the diagrams in Fig. 7. 
pairs with relatively large distances. In contrast, the number of nearest- and next-nearest-neighbor pairs only weakly depends on the shape. Using these correlation functions, we have now defined a mean coordination number for each island: $c_{\lambda}=\sum N(d) \cdot \exp (-\lambda d)$, where $N$ is the number of neighbors with distance $d$. The decay constant $\lambda$ has been determined by searching for a good, linear relationship between $\Delta$ and $c_{\lambda}$. Even for the best $\lambda$ parameter, $10^{-4} \AA^{-1}$, the correlation between gap size and coordination number is poor [Fig. 7(d)]. Apparently, hybridization between the atoms is not the only microscopic origin for a gap closure in the islands, probably because symmetry and shell-closing effects are neglected. Evidently, our empirical eccentricity catches these contributions to the gap size better than the coordination number, and further work is needed to find a quantum mechanical descriptor that mediates geometry and gap size in Au nanostructures.

\section{E. General properties of QWS: Nature of the confinement potential}

So far, we have considered the confinement potential in our Au islands to be infinitely deep, which facilitates the analytical description but oversimplifies the problem. Whereas in bulk gold, the real potential depth for Fermi electrons is given by the workfunction, ${ }^{43}$ this value becomes smaller for $\mathrm{Au}$ islands of finite size. In this last section, we provide a realistic estimation of the potential depth by analyzing the spillout of Au-QWS beyond the physical limits of the islands. The evolution of the density probability $|\psi(x)|^{2}$ with distance from the island edge $x$ is governed by an exponential decay that is controlled by two parameters: $|\psi(x)|^{2} \propto \exp \left[-2 \cdot\left(\alpha_{Q W S}+\alpha_{S T M}\right) \cdot x\right]$. Whereas the STM contribution $\alpha_{S T M}$ accounts for the in-plane decay of the wavefunction overlap between tip and sample, the QWS decay length $\alpha_{Q W S}$ is the consequence of the finite potential depth. The latter provides a direct measure of the true potential height $V_{0}$ according to $\alpha_{Q W S}=\sqrt{\frac{2 m\left(V_{0}-E_{Q W S}\right)}{\hbar^{2}}}$, where $E_{Q W S}$ is the eigenenergy of the respective level as determined from the $d I / d V$ spectra. As our equation contains two unknown variables, $V_{o}$ and $\alpha_{S T M}$, we have analyzed the exponential decay of the $d I / d V$ signal for two independent states, namely, the first and the second QWS of the rectangular island shown in Fig. 3(b). Assuming that the STM decay length $\alpha_{S T M}$ is similar in both cases, we have obtained an estimation for the potential depth experienced by the lowest Au $6 p_{z}$ electrons of $V_{0}=3.75 \mathrm{eV}$. Adding the band onset of $E_{0}=0.75 \mathrm{eV}$, as derived from the dispersion relation, we find an upper edge of the confinement potential at $4.5 \mathrm{eV}$ above the Fermi level, which is smaller than the workfunction of bulk gold $(5.1 \mathrm{eV})$. Two reasons may account for this difference. First, islands in the explored size regime are too small to developed their own vacuum energy and still feel the reduced workfunction of the surrounding $\mathrm{MgO} / \mathrm{Ag}(001)$ film $(3.5 \mathrm{eV}) .{ }^{44}$ Second, the Au electrons may escape the potential well by moving into the $\mathrm{MgO}$ conduction band. In this case, the confinement potential would be given by the oxide electronic structure rather than the vacuum energy.

\section{CONCLUSION}

Using scanning tunneling microscopy and spectroscopy, we have analyzed the electronic structure of planar Au islands containing between 50 and 200 atoms. A series of pronounced $d I / d V$ peaks detected above the Fermi level has been assigned to the manifold of Au $6 p_{z}$ QWS. Surprisingly, their energy position and node structure could be reproduced reasonably well with simple particle-in-a-box models of rectangular, triangular, and linear symmetry. This fair match despite obvious irregularities in the island shape reflects the freeelectron nature of the Au conduction electrons, which renders their properties insensitive to details of the potential. Even better reproduction of the experimental data was achieved by modeling the $\mathrm{Au}$ electronic structure with a parameterized DFTB approach that takes the true atom configuration into account. On the basis of our experimental results, we have established an empirical relationship between the size and shape of the nanoislands and the HOMO-LUMO gap, using the linear eccentricity as a descriptor. With this scheme, we are able to predict the metallicity of supported $\mathrm{Au}$ islands and hence an important factor to evaluate their performance in chemical reactions. We will further exploit this knowledge to investigate the impact of the electronic structure on the adsorption characteristic of metal nanoislands. From the results, we expect deeper insight into the structure-reactivity relationship in heterogeneous catalysis.

\section{ACKNOWLEDGMENTS}

The authors thank Julius Jellinek for insightful discussions. N.N. and H.J.F. are grateful for financial support from the German Research Foundation Excellence Initiative "Unifying Concepts in Catalysis." C.S. thanks the Studienstiftung des Deutschen Volkes for a fellowship. P.K. and H.H. acknowledge support from the Academy of Finland.

\footnotetext{
*Corresponding author: nilius@fhi-berlin.mpg.de

${ }^{1}$ G. Schmid (Ed.), Clusters and Colloids: From Theory to Applications (Wiley-VCH, Weinheim, Germany, 1994).

${ }^{2}$ D. C. Ralph, C. T. Black, and M. Tinkham, Phys. Rev. Lett. 78, 4087 (1997).

${ }^{3}$ A. Bettac, L. Köller, V. Rank, and K. H. Meiwes Broer, Surf. Sci. 402, 475 (1998).

${ }^{4}$ H. Hövel, B. Grimm, M. Bödecker, K. Fieger, and B. Reihl, Surf. Sci. 463, L603 (2000).
}

${ }^{5} \mathrm{X}$. Lin, N. Nilius, H.-J. Freund, M. Walter, P. Frondelius, K. Honkala, and H. Häkkinen, Phys. Rev. Lett. 102, 206801 (2009).

${ }^{6}$ W. A. de Heer, Rev. Mod. Phys. 65, 611 (1993).

${ }^{7}$ U. Landman, B. Yoon, Ch. Zhang, U. Heiz, and M. Arenz, Top. Catal. 44, 145 (2007).

${ }^{8}$ W. Ekardt (Ed.), Metal Clusters (Wiley-VCH, Chichester, UK, 1999).

${ }^{9} \mathrm{H}$. Haberland (Ed.), Clusters of Atoms and Molecules (Springer, Berlin, 1994) 
${ }^{10}$ N. Nilius, T. M. Wallis, and W. Ho, Science 297, 1853 (2002).

${ }^{11}$ S. Fölsch, P. Hyldgaard, R. Koch, and K. H. Ploog, Phys. Rev. Lett. 92, 056803 (2004).

${ }^{12}$ K. F. Braun and K. H. Rieder, Phys. Rev. Lett. 88, 096801 (2002).

${ }^{13}$ J. Kliewer, R. Berndt, and S. Crampin, New J. Phys. 3, 22 (2001).

${ }^{14}$ M. F. Crommie, C. P. Lutz, and D. M. Eigler, Science 262, 218 (1993).

${ }^{15}$ M. Walter, P. Frondelius, K. Honkala, and H. Häkkinen, Phys. Rev. Lett. 99, 096102 (2007).

${ }^{16}$ H. Hövel and I. Barke, New J. Phys. 5, 31 (2003).

${ }^{17}$ J. Li, W. D. Schneider, R. Berndt, and S. Crampin, Phys. Rev. Lett. 80, 3332 (1998).

${ }^{18}$ T. Maltezopoulos, A. Bolz, C. Meyer, C. Heyn, W. Hansen, M. Morgenstern, and R. Wiesendanger, Phys. Rev. Lett. 91, 196804 (2003).

${ }^{19}$ C. Meyer, J. Klijn, M. Morgenstern, and R. Wiesendanger, Phys. Rev. Lett. 91, 076803 (2003).

${ }^{20}$ N. Nilius, Surf. Sci. Reports 64, 595 (2009).

${ }^{21}$ X. Lin, N. Nilius, M. Sterrer, P. Koskinen, H. Häkkinen, and H.-J. Freund, Phys. Rev. B 81, 153406 (2010).

${ }^{22}$ A. Mans, J. H. Dil, A. R. H. F. Ettema, and H. H. Weitering, Phys. Rev. B 66, 195410 (2002).

${ }^{23}$ J. H. Dil, J. W. Kim, S. Gokhale, M. Tallarida, and K. Horn, Phys. Rev. B 70, 045405 (2004).

${ }^{24}$ N. Nilius, M. V. Ganduglia-Pirovano, V. Brazdova, M. Kulawik, J. Sauer, and H. J. Freund, Phys. Rev. Lett. 100, 096802 (2008).

${ }^{25}$ M. Haruta, Cattech 6, 102 (2002).

${ }^{26}$ M. S. Chen and D. W. Goodman, Science 306, 252 (2004).

${ }^{27}$ U. Kreibig and W. Vollmer (Eds.), Optical Properties of Metal Clusters (Springer, Berlin, 1995).

${ }^{28}$ P. Koskinen and V. Mäkinen, Comput. Mat. Sci. 47, 237 (2009).

${ }^{29}$ J. Tersoff and D. R. Hamann, Phys. Rev. Lett. 50, 1998 (1983).
${ }^{30}$ S. Schintke, S. Messerli, M. Pivetta, F. Patthey, L. Libioulle, M. Stengel, A. DeVita, and W. D. Schneider, Phys. Rev. Lett. 87, 276801 (2001)

${ }^{31}$ M. Sterrer, T. Risse, M. Heyde, H.-P. Rust, and H.-J. Freund, Phys. Rev. Lett. 98, 206103 (2007).

${ }^{32}$ G. Pacchioni, L. Giordano, and M. Baistrocchi, Phys. Rev. Lett. 94, 226104 (2005)

${ }^{33}$ D. Ricci, A. Bongiorno, G. Pacchioni, and U. Landman, Phys. Rev. Lett. 97, 036106 (2006).

${ }^{34}$ V. Mäkinen, P. Koskinen, and H. Häkkinen, Eur. Phys. J. D 67, 38 (2013); P. Koskinen, H. Häkkinen, G. Seifert, S. Sanna, T. Frauenheim, and M. Moseler, New J. Phys. 8, 9 (2006).

${ }^{35}$ V. Simic-Milosevic, M. Heyde, X. Lin, T. König, H.-P. Rust, M. Sterrer, T. Risse, N. Nilius, H.-J. Freund, L. Giordano, and G. Pacchioni, Phys. Rev. B 78, 235429 (2008).

${ }^{36}$ N. Nilius, M. V. Ganduglia-Pirovano, V. Brazdova, M. Kulawik, J. Sauer, and H. J. Freund, Phys. Rev. B 81, 045422 (2010).

${ }^{37}$ J. Repp, G. Meyer, S. Paavilainen, F. E. Olsson, and M. Persson, Phys. Rev. Lett. 95, 225503 (2005).

${ }^{38}$ D. J. Griffiths (Ed.), Introduction to Quantum Mechanics (Prentice Hall, Upper Saddle River, New Jersey, 2004).

${ }^{39}$ S. LaShell, B. A. McDougall, and E. Jensen, Phys. Rev. Lett. 77, 3419 (1996).

${ }^{40}$ W. K. Li, J. Chem. Edu. 61, 1034 (1984).

${ }^{41}$ The $\Delta$ values may differ with respect to earlier publications due to a different definition of the band gap. Whereas in Ref. 5, $\Delta$ values were estimated directly from the zero-bias gap in the $d I / d V$ spectra, the gap size here is defined as distance between HOMO and LUMO level, derived from a deconvolution into Gauss peaks.

${ }^{42}$ U. Röthlisberger and W. Andreoni, J. Chem. Phys. 94, 8129 (1991).

${ }^{43}$ D. R. Lide (Ed.), CRC Handbook of Chemistry and Physics (CRC Press, Boca Raton, Louisiana, 2010).

${ }^{44}$ T. König, G. H. Simon, H.-P. Rust, and M. Heyde, J. Phys. Chem. C 113, 11301 (2009). 